\title{
Dust evolution with active galactic nucleus feedback in elliptical galaxies
}

\author{
Hiroyuki Hirashita ${ }^{a}$, Takaya Nozawa ${ }^{b}$ \\ ${ }^{a}$ Institute of Astronomy and Astrophysics, Academia Sinica, P.O. Box 23-141, Taipei 10617, Taiwan \\ ${ }^{b}$ National Astronomical Observatory of Japan, Mitaka, Tokyo 181-8588, Japan
}

\begin{abstract}
We have recently suggested that dust growth in the cold gas phase dominates the dust abundance in elliptical galaxies while dust is efficiently destroyed in the hot X-ray emitting plasma (hot gas). In order to understand the dust evolution in elliptical galaxies, we 'construct a simple model that includes dust growth in the cold gas and dust destruction in the hot gas. We also take into account the effect of mass exchange between these two gas components induced by active galactic nucleus (AGN) feedback. We survey reasonable ranges of the relevant parameters in the model and find that AGN feedback cycles actually produce a variety in cold gas mass and dust-to-gas ratio. By comparing with an observational sample of nearby elliptical galaxies, we find that, although the dust-to-gas ratio varies by an order of magnitude in our model, the entire range of the observed dust-to-gas ratios is difficult to be reproduced under a single parameter set. Variation of the dust growth efficiency is the most probable solution to explain the large variety in dust-to-gas ratio of the observational sample. Therefore, dust growth can play a central role in creating the variation in dust-to-gas ratio through the AGN feedback cycle and through the variation in dust growth efficiency.
\end{abstract}

Keywords: Active galactic nuclei, Dust, Elliptical galaxies, Galaxy evolution, Interstellar medium

\section{Introduction}

In the nearby Universe, elliptical galaxies are known to have less gas, dust, and ongoing star formation activity than spiral 'galaxies. Yet, they still have some amount of interstellar gas in the form of hot X-ray-emitting halo gas (e.g., O’Sullivan et al., 2001) and cold gas (e.g., Wiklind et al., 1995). Moreover, dust is detected for a significant fraction of elliptical galaxies by optical extinction (e.g., Goudfrooij et al., 1994; van Dokkum and Franx, 1995; Ferrari et al., 1999; Tran et al., 2001) or far-infrared (FIR) emission (e.g., Knapp et al., 1989; Smith et al., 2012; di Serego Alighieri et al., 2013). Dust mass is estimated from the reddening in the optical or from FIR dust emission, and ranges from $\sim 10^{4}$ to $\sim 10^{7} M_{\odot}$ (e.g., Goudfrooij et al., 1994; Leeuw et al., 2004). Since the existence of dust could affect the cooling and chemical processes (Dwek, 1987; Fabian et al., 1994; Voit and Donahue, 1995; Seok et al., 2015; Hirashita et al., 2015), the understanding of the origin and evolution of dust in elliptical galaxies is important in clarifying their evolution.

Because the stellar population is dominated by old stars whose ages are comparable to the cosmic age, the dust in elliptical galaxies is predominantly supplied by asymptotic giant branch (AGB) stars rather than by supernovae. However, dust destruction by sputtering in the X-ray emitting plasma is so efficient that the observed dust mass cannot be explained by the balance between the supply from AGB stars and the destruction (e.g., Patil et al., 2007). Thus, some authors argue that the

Email address: hirashita@asiaa.sinica.edu.tw (Hiroyuki Hirashita) dust existing in elliptical galaxies is possibly injected from outside via the merging or accretion of external galaxies (Forbes, 1991; Temi et al., 2004; Fujita et al., 2013). The lack of correlation between dust FIR luminosity and stellar luminosity is also taken as evidence of external origin of dust (Temi et al., 2007); however, this argument may not hold if dust is processed by mechanisms unrelated to stars.

Recently, Hirashita et al. (2015) have proposed that the existence of dust in elliptical galaxies can be explained by dust growth by the accretion of gas phase metals in the cold interstellar medium (ISM). They also suggest that the presence of dust growth also explains the extinction curves observed in elliptical galaxies. The dust-to-gas ratio could become as high as $\gtrsim 10^{-3}$ by accretion, explaining the high dust abundance in some elliptical galaxies. However, dust growth in the cold gas has not been considered as a major source of dust in the context of dust evolution in elliptical galaxies. Dust growth has already been noted as a major mechanism of dust mass increase in a wide context of galaxy evolution (e.g., Dwek, 1998; Hirashita, 1999; Inoue, 2003; Zhukovska et al., 2008; Valiante et al., 2011; Mattsson and Andersen, 2012; Mancini et al., 2015; Popping et al., 2016; Hou et al., 2016; Zhukovska et al., 2016), and some experimental studies have also shown that dust grains could grow by accreting the gasphase metals (Rouillé et al. 2014; but see Ferrara et al. 2016). Thus, constructing a dust evolution model that includes this dust growth mechanism would contribute to the understanding of dust evolution in elliptical galaxies.

The overall evolution of dust should be considered in relation to the gas evolution, especially because gas and dust are usually dynamically coupled on galactic scales. Recent studies have 
proposed that the gas cooling in galaxies is strongly regulated by the energy input from active galactic nuclei (AGNs). The energy input from AGN winds (Silk and Rees, 1998; Fabian, 1999; King, 2005) or AGN jets (Wagner and Bicknell, 2011; Mukherjee et al., 2016) prevents cooling flows from occurring and/or makes cold gas evaporate (see also Ciotti and Ostriker, 2001; Fabian, 2012). These kinds of energy input are called AGN feedback, and are considered to play an important role in galaxy formation and evolution (Croton et al., 2006; Booth and Schave, 2009)

Temi et al. (2007) showed the existence of a dust emission component extended over $5-10 \mathrm{kpc}$ in elliptical galaxies using the Spitzer $70 \mu \mathrm{m}$ band data. They suggest that this cold dust component was originally contained in the cold gas, which was then heated by AGN feedback and eventually mixed with the hot gas. In their scenario, the heated gas is transported into the hot gas by buoyant force, and the time-scale of the transport is around $10^{7}$ yr. Kaneda et al. (2011) also showed in an elliptical galaxy (NGC 4125) a dust emission component whose extension is similar to the distribution of the hot X-ray emitting halo. The multi-phase structures and irregular morphologies of X-ray emitting hot plasmas could be explained by bubbles created by AGN feedback (Buote et al., 2003).

A part of the hot gas may cool down to reform the cold gas, which could contribute to the fueling of AGN (Werner et al., 2014). AGN feedback may compress the surrounding gas and enhance gas cooling locally (Valentini and Brighenti, 2015). Because dust grains can grow in the cold gas as mentioned above, the cold gas injected into the hot gas by AGN feedback would supply the dust to the hot gas. This dust supply could be dominant over the dust production by AGB stars. If so, formation of cold gas and the subsequent AGN fueling leading to AGN feedback play a dominant role in the dust evolution in elliptical galaxies.

If the dust evolution is affected by episodic AGN activities, which appear as a result of a cycle of gas ejection and cooling, the statistical properties of the dust abundances in elliptical galaxies are determined by the time-scales of dust processing relative to the period of an AGN cycle. Lauer et al. (2005) inferred the period of an AGN cycle in early-type galaxies based on the dust lifetime against sputtering in the hot gas and the fraction of dust detection for a sample, obtaining a period of $\sim 10^{8} \mathrm{yr}$. The dust contained in the cold gas may be injected into the hot gas in an episodic way associated with the AGN cycle, if AGN feedback efficiently heats the cold gas (Mathews et al., 2013).

In this paper, we make a theoretical model of dust evolution in AGN feedback cycles by including important physical processes such as the mass exchange between the hot and cold gas components and the dust evolution in those gases. For dust processing, we consider dust destruction by sputtering in the hot gas and dust growth by the accretion of gas-phase metals in the cold gas. This modeling enables us to examine how AGN feedback cycles affect the dust abundances in elliptical galaxies. We can also examine the effect of dust growth on the dust abundance in elliptical galaxies for the first time.

The paper is organized as follows: we formulate the model of dust evolution in an elliptical galaxy in Section 2. We show the results in Section 3. We discuss the model predictions, and compare them with observational data in Section 4 Finally we conclude in Section 5

\section{Model}

We construct a model that describes the dust evolution in an elliptical galaxy. For dust sources, in addition to AGB stars considered in previous studies (Mathews and Brighenti, 2003; Patil et al., 2007), we also consider dust supply from the cold gas where dust grows by the accretion of gas-phase metals. The dust in the cold gas is injected into the hot gas by AGN feedback together with the cold gas. The dust injected into the hot gas is destroyed by sputtering. The hot component $\left(\sim 10^{7} \mathrm{~K}\right)$ is the gas whose temperature is comparable to the virial temperature determined by the global gravitational potential of the elliptical galaxy while the cold component is cold and dense enough to host dust growth ( $\lesssim 100 \mathrm{~K}$; Hirashita et al., 2015). To make the model as simple as possible, we treat each gas component as a single zone and consider the mass exchange between the two components. Dust growth and destruction are treated consistently with the evolution of each gas component as explained below.

\subsection{Basic equations}

We consider the mass exchange between the hot and cold phases. The evolution of the cold gas mass $M_{\mathrm{g}, \mathrm{c}}$ as a function of time $t$ is written as

$\frac{d M_{\mathrm{g}, \mathrm{c}}}{d t}=\dot{M}_{\mathrm{in}}-\dot{M}_{\mathrm{ret}}$,

where $\dot{M}_{\text {in }}$ is the infall rate of the cooled gas and $\dot{M}_{\text {ret }}$ is the gas return rate from the cold to the hot phase by AGN feedback. These two terms are modeled below.

The hot gas is treated as a constant reservoir of gas for simplicity; that is, we assume that the mass of hot gas, $M_{\mathrm{g}, \mathrm{h}}$ is constant $\left(d M_{\mathrm{g}, \mathrm{h}} / d t=0\right)$. This treatment neglects the complication arising from the possibility that there could be a supply/loss of hot gas from/to outside. Because the hot gas basically acts as an efficient destroyer of dust regardless of the choice of $M_{\mathrm{g}, \mathrm{h}}$, the value of $M_{\mathrm{g}, \mathrm{h}}$ has a minor influence on the results compared with other parameters. However, we should note that the dustto-gas ratio in the hot gas is directly affected by $M_{\mathrm{g}, \mathrm{h}}$ since it directly enters the dust-to-gas ratio in the hot gas. We discuss the value of $M_{\mathrm{g}, \mathrm{h}}$ again in Section 2.3 .

The time evolution of the dust mass in the cold phase, $M_{\mathrm{d}, \mathrm{c}}$, is written as

$$
\frac{d M_{\mathrm{d}, \mathrm{c}}}{d t}=\mathcal{D}_{\mathrm{h}} \dot{M}_{\mathrm{in}}-\mathcal{D}_{\mathrm{c}} \dot{M}_{\mathrm{ret}}+\frac{M_{\mathrm{d}, \mathrm{c}}}{\tau_{\text {grow }}},
$$

where $\mathcal{D}_{\mathrm{h}} \equiv M_{\mathrm{d}, \mathrm{h}} / M_{\mathrm{g}, \mathrm{h}}$ and $\mathcal{D}_{\mathrm{c}} \equiv M_{\mathrm{d}, \mathrm{c}} / M_{\mathrm{g}, \mathrm{c}}$ are the dust-to-gas ratios in the hot and cold phases, respectively $\left(M_{\mathrm{d}, \mathrm{h}}\right.$ and $M_{\mathrm{d}, \mathrm{c}}$ are the dust mass in the hot and cold gas, respectively), and $\tau_{\text {grow }}$ is the time-scale of dust growth by the accretion of gas-phase metals in the cold gas. The dust growth timescale $\tau_{\text {grow }}$ is given 
Table 1: Parameters in the model.

\begin{tabular}{lccc}
\hline Parameter & Unit & Fiducial $^{a}$ & Range $^{b}$ \\
\hline$\dot{M}_{\text {in }, 0}$ & $M_{\odot} \mathrm{yr}^{-1}$ & 3 & $1-10$ \\
$\tau_{\text {tr }}$ & $\mathrm{yr}$ & $10^{7}$ & $(0.3-3) \times 10^{7}$ \\
$\tau_{\text {AGN }}$ & $\mathrm{yr}$ & $10^{8}$ & fixed \\
$f_{\text {ret }}$ & - & 0.3 & $0.1-0.5$ \\
$\tau_{\text {acc }}$ & $\mathrm{yr}$ & $10^{7}$ & $10^{6}-10^{8}$ \\
$\tau_{\text {sput }}$ & $\mathrm{yr}$ & $10^{7}$ & $10^{6}-10^{8}$ \\
$Z$ & - & 0.02 & fixed \\
$\mathcal{D}_{\text {AGB }}$ & - & 0.01 & fixed \\
$\alpha$ & $\mathrm{yr}^{-1}$ & $1.6 \times 10^{-12}$ & fixed \\
$M_{*}$ & $M_{\odot}$ & $10^{10.5}$ & $10^{10}-10^{11}$ \\
\hline
\end{tabular}

${ }^{a}$ Fiducial value.

${ }^{b}$ Range of values investigated unless the parameter value is fixed.

in Section 2.3 (equation 9), and is treated as a function of $\mathcal{D}_{\mathrm{c}}$. The time evolution of the dust mass in the hot phase, $M_{\mathrm{h}, \mathrm{c}}$, on the other hand, is written as

$\frac{d M_{\mathrm{d}, \mathrm{h}}}{d t}=-\mathcal{D}_{\mathrm{h}} \dot{M}_{\mathrm{in}}+\mathcal{D}_{\mathrm{c}} \dot{M}_{\mathrm{ret}}-\frac{M_{\mathrm{d}, \mathrm{h}}}{\tau_{\text {sput }}}+\mathcal{D}_{\mathrm{AGB}} \alpha M_{*}$,

where $\tau_{\text {sput }}$ is the dust destruction time-scale by sputtering in the hot gas, $\mathcal{D}_{\mathrm{AGB}}$ is the dust-to-gas ratio in AGB star winds, $\alpha$ is the mass loss rates of AGB stars per stellar mass, and $M_{*}$ is the total stellar mass $\left(\alpha M_{*}\right.$ is the total mass loss rate of AGB stars). Using $M_{\mathrm{d}, \mathrm{c}}=\mathcal{D}_{\mathrm{c}} M_{\mathrm{g}, \mathrm{c}}, M_{\mathrm{d}, \mathrm{h}}=\mathcal{D}_{\mathrm{h}} M_{\mathrm{g}, \mathrm{h}}$, and equation (1), we rewite equations (2) and (3) as the following equations for the dust-to-gas ratios:

$\frac{d \mathcal{D}_{\mathrm{c}}}{d t}=\frac{\mathcal{D}_{\mathrm{c}}}{\tau_{\text {grow }}}+\left(\mathcal{D}_{\mathrm{h}}-\mathcal{D}_{\mathrm{c}}\right) \frac{\dot{M}_{\mathrm{in}}}{M_{\mathrm{g}, \mathrm{c}}}$,

$\frac{d \mathcal{D}_{\mathrm{h}}}{d t}=-\mathcal{D}_{\mathrm{h}} \frac{\dot{M}_{\mathrm{in}}}{M_{\mathrm{g}, \mathrm{h}}}-\frac{\mathcal{D}_{\mathrm{h}}}{\tau_{\mathrm{sput}}}+\mathcal{D}_{\mathrm{c}} \frac{\dot{M}_{\mathrm{ret}}}{M_{\mathrm{g}, \mathrm{h}}}+\mathcal{D}_{\mathrm{AGB}} \frac{\alpha M_{*}}{M_{\mathrm{g}, \mathrm{h}}}$.

We solve equations (1), (4) and (5). Below we formulate some undetermined terms and estimate a reasonable range for each parameter (Table 1 ).

\subsection{Mass exchange between the phases}

The mass exchange between the cold and hot phases is described by $\dot{M}_{\text {in }}$ (inflow of cooled hot gas to the cold gas) and $\dot{M}_{\text {ret }}$ (return of cold gas to the hot phase by AGN feedback). For simplicity, we assume that AGN feedback occurs periodically with a period of $\tau_{\mathrm{AGN}}$. During an episode of AGN feedback, the cold gas is transported to the hot gas, and this transportation time-scale is parameterized by $\tau_{\mathrm{tr}}$. This transport (or the return of cold gas to the hot gas) lasts for a fraction of $\tau_{\mathrm{AGN}}$, and this fraction is parameterized by $f_{\text {ret. }}$. That is, the duration of an AGN feedback episode is $f_{\text {ret }} \tau_{\mathrm{AGN}}$. We assume that the outflow $\left(\dot{M}_{\text {ret }}\right)$ of the cold gas is on during the AGN feedback as

$\dot{M}_{\text {ret }}(t)= \begin{cases}M_{\mathrm{g}, \mathrm{c}} / \tau_{\mathrm{tr}} & \text { if } 0 \leq t / \tau_{\mathrm{AGN}}-\left[t / \tau_{\mathrm{AGN}}\right] \leq f_{\mathrm{ret}} \\ 0 & \text { otherwise }\end{cases}$ where $[x]$ is the floor function, which indicates the largest integer that satisfies $[x] \leq x$. This equation indicates that the outflow is periodically turned on every $\tau_{\mathrm{AGN}}$ with a duration of $f_{\text {ret }} \tau_{\text {AGN }}$.

The inflow of cooled hot gas is assumed to occur only when there is no outflow activity (i.e., when $M_{\text {ret }}=0$ ):

$\dot{M}_{\mathrm{in}}(t)= \begin{cases}0 & \text { if } 0 \leq t / \tau_{\mathrm{AGN}}-\left[t / \tau_{\mathrm{AGN}}\right] \leq f_{\mathrm{ret}}, \\ \dot{M}_{\mathrm{in}, 0} & \text { otherwise, }\end{cases}$

where we treat $\dot{M}_{\text {in, } 0}$ as a constant free parameter.

Based on the inferred dust lifetime and dynamical time in the center of an elliptical galaxy and the rate of dust detection for elliptical galaxies, Lauer et al. (2005) suggested that the cycle of dust appearance and disappearance is $\sim 10^{8} \mathrm{yr}$. We can regard this time as $\tau_{\mathrm{AGN}}$ if that dust cycle is induced by the AGN feedback cycle. Thus, we adopt $\tau_{\mathrm{AGN}}=10^{8} \mathrm{yr}$. Since the time evolution of gas and dust is determined by the time-scale relative to $\tau_{\mathrm{AGN}}$, we fix $\tau_{\mathrm{AGN}}$ and change other time-scales as described below. The maximum cold gas mass is roughly estimated as $\dot{M}_{\text {in }, 0} \tau_{\text {AGN }}\left(1-f_{\text {ret }}\right)$, which should be comparable to the observed gas mass $\sim$ a few $\times 10^{8} M_{\odot}$ (e.g., Wiklind et al., 1995). This means that $\dot{M}_{\text {in }, 0} \sim$ a few $\times\left(1-f_{\text {ret }}\right)^{-1} M_{\odot} \mathrm{yr}^{-1}$. Thus, we investigate a range of $\sim 1-10 M_{\odot} \mathrm{yr}^{-1}$. Since cold gas is detected in a significant fraction of elliptical galaxies, $f_{\text {ret }}$ cannot be very near to 1 . Thus, we focus on a range of $0<f_{\text {ret }} \leq 0.5$.

For $\tau_{\text {tr }}$, it may be reasonable to adopt the dynamical time. As mentioned in the Introduction, Temi et al. (2007) suggested dust transport by buoyancy acting on the heated cold gas in order to explain the origin of extended dust FIR emission component. They estimated the time-scale of transport as $\sim 10^{7} \mathrm{yr}$ based on the dynamical time-scale. Thus, we adopt $\tau_{\operatorname{tr}} \sim 10^{7}$ $\mathrm{yr}$, and also examine an order-of-magnitude range for $\tau_{\mathrm{tr}}$.

\subsection{Parameters for dust evolution}

We have introduced two time-scale parameters that govern the dust evolution: dust growth time-scale $\left(\tau_{\text {grow }}\right)$ and dust destruction (sputtering) time-scale $\left(\tau_{\text {sput }}\right)$.

We adopt the following estimate of the time-scale of grain growth (Asano et al., 2013):

$$
\begin{aligned}
\tau_{\text {acc }}= & 2 \times 10^{6}\left(\frac{a}{0.1 \mu \mathrm{m}}\right)\left(\frac{n_{\mathrm{H}, \mathrm{c}}}{10^{3} \mathrm{~cm}^{-3}}\right)^{-1}\left(\frac{T_{\mathrm{gas}}}{50 \mathrm{~K}}\right)^{-1 / 2} \\
& \times\left(\frac{Z}{0.02}\right)^{-1} \mathrm{yr},
\end{aligned}
$$

where $a$ is the grain radius, $n_{\mathrm{H}, \mathrm{c}}$ is the hydrogen number density in the cold gas, $T_{\text {gas }}$ is the gas temperature, and $Z$ is the metallicity (e.g., Inoue, 2011; Asano et al., 2013). Because of some poorly constrained parameters such as $a$ and $n_{\mathrm{H}, \mathrm{c}}$, we treat $\tau_{\text {acc }}$ as a given constant parameter for simplicity, but investigate a wide range in $\tau_{\text {acc }}$. Moreover, since accretion is efficient only in the dense medium, it depends on the mass fraction of dense gas in the cold gas, which is denoted as $f_{\text {dense. The dust }}$ growth time-scale is effectively estimated as $\tau_{\text {acc }} / f_{\text {dense }}$. Based on the above estimate, we examine $\tau_{\text {acc }}=10^{6}-10^{8} \mathrm{yr}\left(10^{7} \mathrm{yr}\right.$ for the fiducial case), allowing for a small value for $f_{\text {dense }}$ or variation in $a$ and $n_{\mathrm{H}, \mathrm{c}}$ (Kuo et al., 2013; Schneider et al., 2016; 
Aoyama et al., 2017). In reality, the accretion time-scale is also affected by grain size distribution (Kuo and Hirashita, 2012); thus, the above $a$ represents the appropriate average of the grain size 1 Considering the uncertainties in grain size distribution, $\tau_{\text {acc }}$ may be out of the range above $\left(10^{6}-10^{8} \mathrm{yr}\right)$. If $\tau_{\text {acc }} \lesssim 10^{6}$ $\mathrm{yr}$, the grain growth is so efficient that the dust-to-gas ratio in the cold gas stays at a maximum value given below $\left(\mathcal{D}_{\text {sat }}\right)$ for most of the time regardless of the value of $\tau_{\text {acc }}\left(\lesssim 10^{6} \mathrm{yr}\right)$. If $\tau_{\text {acc }} \gtrsim 10^{8} \mathrm{yr}$, in contrast, dust growth is not efficient enough to raise the dust-to-gas ratio above $10^{-4}$. Therefore, the range of $\tau_{\text {acc }}=10^{6}-10^{8} \mathrm{yr}$ covers all the resulting behavior of interest for $\mathcal{D}_{c}$, i.e., covers all the interesting range of $\mathcal{D}_{\mathrm{c}}$ as we show below.

Using a given value of $\tau_{\text {acc }}$, the dust growth time-scale is given by

$\tau_{\text {grow }}=\frac{\tau_{\text {acc }}}{1-\mathcal{D}_{\mathrm{c}} / \mathcal{D}_{\text {sat }}}$,

where the denominator on the right-hand side means that accretion is saturated as $\mathcal{D}_{\mathrm{c}}$ approaches $\mathcal{D}_{\text {sat }}$, which is the abundance of the metals available for dust formation. We assume $\mathcal{D}_{\text {sat }}=0.01$ in this paper, since we are considering solar metallicity environment (i.e., $\mathcal{D}_{\text {sat }} \sim Z_{\odot}$ ). The abundance of available metals for dust formation may be correlated with the stellar mass because of the stellar mass-metallicity relation (Gallazzi et al., 2005). However, this relation has a large scatter, and it is also suggested that the velocity dispersion (or the virial mass) is more tightly related to the stellar metallicity (Smith et al., 2009). Moreover, it is not clear how tightly the stellar metallicity is related to the gas metallicity because of accretion of external gas (Su and Irwin, 2013). Thus, we simply adopt a constant $\mathcal{D}_{\text {sat }}$, but we also note that increasing/decreasing $\mathcal{D}_{\text {sat }}$ has broadly the same effect of short/long $\tau_{\text {acc }}$.

The time-scale of dust destruction by sputtering is estimated as (Tsai and Mathews, 1995; Nozawa et al., 2006; Hirashita et al., 2015)

$\tau_{\text {sput }}=7.1 \times 10^{6}\left(\frac{a}{0.1 \mu \mathrm{m}}\right)\left(\frac{n_{\mathrm{H}, \mathrm{h}}}{10^{-2} \mathrm{~cm}^{-3}}\right)^{-1} \mathrm{yr}$,

where $n_{\mathrm{H}, \mathrm{h}}$ is the number density of hydrogen nuclei in the hot gas. As we did for $\tau_{\text {acc }}$ above, we treat $\tau_{\text {sput }}$ as a given constant parameter. Based on this estimation, we adopt $\tau_{\text {sput }}=10^{7} \mathrm{yr}$ for the fiducial case and examine a range of $10^{6}-10^{8} \mathrm{yr}$ considering the variety in $a$ and $n_{\mathrm{H}, \mathrm{h}}$. Note that fixing $\tau_{\text {acc }}$ and $\tau_{\text {sput }}$ implicitly assumes a fixed grain size (or grain size distribution). In reality, the grain size distribution itself is determined as a result of the cycle of dust destruction and growth. Therefore, a consistent treatment of those time-scales and the grain size distribution would be necessary. Hirashita et al. (2015) considered the evolution of grain size distribution by dust destruction and subsequent dust growth and showed that the resulting grain

${ }^{1}$ More precisely, $a$ is the ratio of the mean $a^{3}$ to the mean $a^{2}$ (Hirashita and Kuo, 2011). As shown in Hirashita and Kuo (2011), the time variation of $a$ could be effectively incorporated in the definition of $\tau_{\text {acc }}$ so that $\tau_{\text {acc }}$ could still be treated as constant. size distribution is consistent with the optical extinction curves observed by Patil et al. (2007), although they did not consider a full cycle of AGN feedback. At the same time, they also showed that the resulting grain size distribution depends on the size distribution of dust grains produced by AGB stars. To avoid such a complication, we treat $\tau_{\text {acc }}$ and $\tau_{\text {sput }}$ as given constant parameters and concentrate on the total dust mass. The grain size distribution realized after AGN feedback cycles will be investigated in the future work.

We adopt $\mathcal{D}_{\mathrm{AGB}}=0.01, \alpha=1.6 \times 10^{-12} \mathrm{yr}^{-1}$, and $M_{*}=$ $10^{10}-10^{11} M_{\odot}$ (Tsai and Mathews, 1995). However, the precise values of these quantities do not affect the dust abundance in the cold gas as long as the dust formed in AGB stars is efficiently destroyed by the hot gas. We fix $\mathcal{D}_{\mathrm{AGB}}$ and $\alpha$, and only focus on the variation of $M_{*}$.

As explained in Section 2.1 we fix the hot gas mass $M_{\mathrm{g}, \mathrm{h}}$ for simplicity. The hot gas mass in the central a few kpc, which could directly exchange the gas mass with the central cold component, is estimated as $10^{8}-10^{9} M_{\odot}$ (Tsai and Mathews, 1995). Thus, we adopt $M_{\mathrm{g}, \mathrm{h}}=3 \times 10^{8} M_{\odot}$. The dust-to-gas ratio in the hot gas $\left(\mathcal{D}_{\mathrm{h}}\right)$ is inversely proportional to $M_{\mathrm{g}, \mathrm{h}}$ by definition; however, because dust in the hot gas is efficiently destroyed by sputtering, as shown below, $\mathcal{D}_{\mathrm{h}} \ll \mathcal{D}_{\mathrm{c}}$ is satisfied when the hot gas is actively accreted on to the cold gas, except for the case where the sputtering time-scale is as long as the AGN feedback cycle $\left(\tau_{\text {sput }} \gtrsim \tau_{\text {AGN }}\right)$. Therefore, the inflow is practically a "dust-free" gas for the cold gas, so that the value of $\mathcal{D}_{\mathrm{h}}$ does not influence $\mathcal{D}_{\mathrm{c}}$. This means that the choice of $M_{\mathrm{g}, \mathrm{h}}$, which could affect $\mathcal{D}_{\mathrm{h}}$, does not have a significant influence on $\mathcal{D}_{\mathrm{c}}$.

We assume the following initial conditions: $\mathcal{D}_{\mathrm{c}}=\mathcal{D}_{\mathrm{h}}=$ $10^{-6}$, and $M_{\mathrm{g}, \mathrm{c}}=10^{7} M_{\odot}$. As seen later, the initial condition is not important, since the system "forgets" the initial condition in $\sim \tau_{\mathrm{AGN}}$.

\section{Results}

\subsection{Fiducial case}

First of all, we show the time evolution of relevant quantities under the fiducial parameter values listed in Table 1, In Fig. 1, we show the evolutions of the dust-to-gas ratios in the hot and cold gas, the cold gas mass, and the dust masses in the cold and hot gas. Here we describe how each of these quantities evolves as a function of time.

All the quantities have almost periodic behaviors with a period given by $\tau_{\mathrm{AGN}}$. In association with the onset of AGN feedback, the dust grown in the cold gas is injected into the hot gas, so that the dust-to-gas ratio in the hot gas increases. However, the dust supplied to the hot gas is quickly destroyed by sputtering, and the dust-to-gas in the hot gas tends to converge to the value determined by the balance between dust supply from stars and dust destruction by sputtering. After AGN feedback stops, the cold gas increases its mass by gas infall. In this phase, because the dust-to-gas ratio in the infalling gas, which is equal to the dust-to-gas ratio in the hot gas, is smaller than that in the cold gas, the dust-to-gas ratio in the cold gas decreases as a result of dilution. 


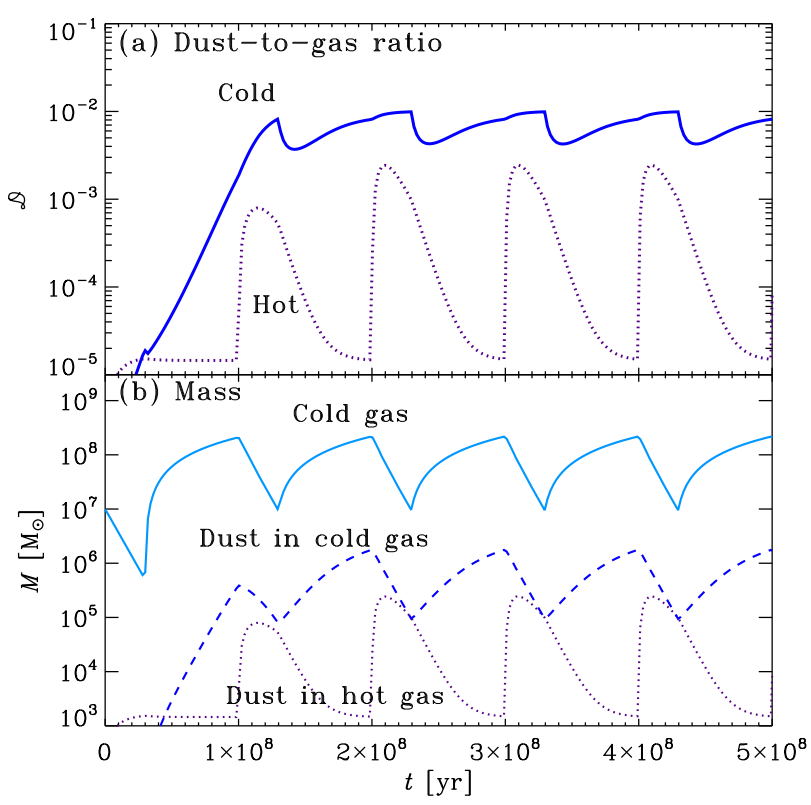

Figure 1: Time evolutions of relevant quantities. (a) Dust-to-gas ratio in the cold $\left(\mathcal{D}_{\mathrm{c}}\right)$ and hot gas $\left(\mathcal{D}_{\mathrm{h}}\right)$ (solid and dotted lines, respectively). (b) Cold gas mass $\left(M_{\mathrm{g}, \mathrm{c}}\right)$ (solid line), and dust masses in the cold gas $\left(M_{\mathrm{d}, \mathrm{c}}\right)$ (dashed line) and in the hot gas $\left(M_{\mathrm{h}, \mathrm{c}}\right)$ (dotted line).

The above general behavior does not change by the choice of parameter values. Thus, for the purpose of simplification, we hereafter concentrate on the dust-to-gas ratios in the cold and hot gas $\left(\mathcal{D}_{\mathrm{c}}\right.$ and $\left.\mathcal{D}_{\mathrm{h}}\right)$, and the cold gas mass $\left(M_{\mathrm{g}, \mathrm{c}}\right)$, since the dust masses in the hot and cold gas just trace the evolution of the corresponding dust-to-gas ratios. Below we show the dependence on each parameter listed in Table 1.

\subsection{Mass inflow rate}

We consider the mass accretion onto the cold gas when AGN feedback is off. The mass inflow rate is regulated by the parameter $\dot{M}_{\mathrm{in}, 0}$. In Fig. 2 we show the time evolution of $\mathcal{D}_{\mathrm{c}}, \mathcal{D}_{\mathrm{h}}$, and $M_{\mathrm{g}, \mathrm{c}}$ for $\dot{M}_{\mathrm{in}, 0}=3$ (fiducial), 1 , and $10 M_{\odot} \mathrm{yr}^{-1}$. Below we describe how the results depend on $\dot{M}_{\text {in }, 0}$.

The dust-to-gas ratio in the hot gas has a larger amplitude for a larger $\dot{M}_{\mathrm{in}, 0}$. This is because more dust is accumulated in the cold gas. When this dusty cold gas is injected into the hot gas by AGN feedback, the hot gas is enriched with dust. The dust injected into the hot gas is readily destroyed by sputtering; thus, the hot gas is dust-poor for most of the time. The dustto-gas ratio in the hot gas drops slightly more in the case of a higher $M_{\mathrm{in}, 0}$ because the inflow transports the dust from the hot gas to the cold gas in our model. However, the lowest level of the dust-to-gas ratio in the hot gas is not very sensitive to $M_{\mathrm{in}, 0}$ since it is determined by the balance between dust supply from AGB stars and dust destruction by sputtering. In terms of the dust-to-gas ratio in the cold gas, the periodic decrease is due to the inflow of dust-poor gas. However, since dust growth is efficient enough, the original high dust-to-gas ratio is recovered as soon as the cold gas attains enough mass by the inflow. As

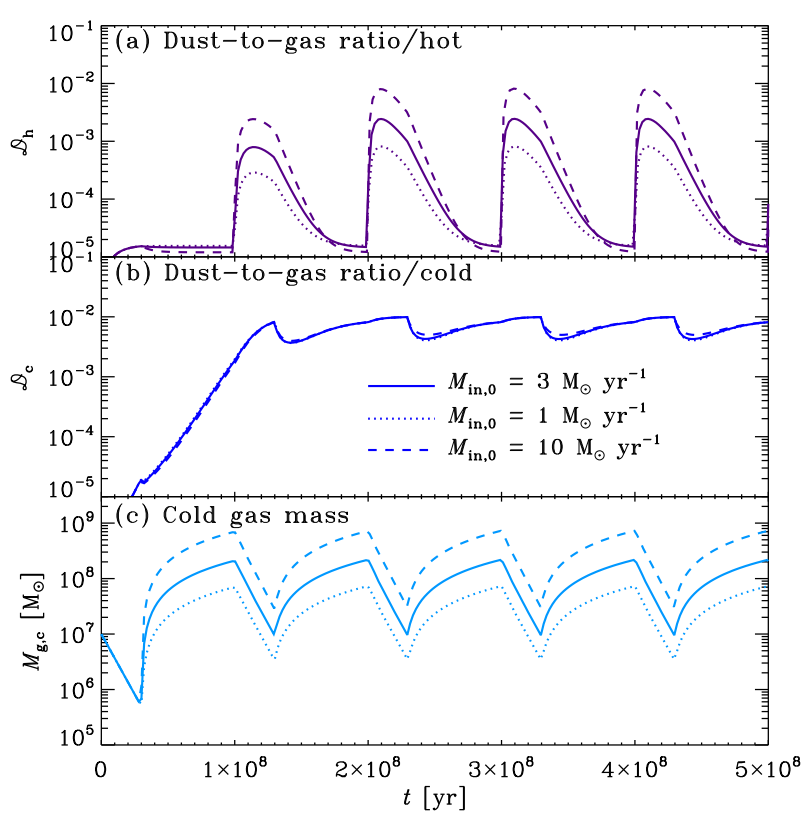

Figure 2: Time evolutions of relevant quantities. (a) Dust-to-gas ratio in the hot gas $\left(\mathcal{D}_{\mathrm{h}}\right)$. (b) Dust-to-gas ratio in the cold gas $\left(\mathcal{D}_{\mathrm{c}}\right)$. (c) Cold gas mass $\left(M_{\mathrm{g}, \mathrm{c}}\right)$. The solid, dotted, and dashed lines show the results for $\dot{M}_{\mathrm{in}, 0}=3$ (fiducial), 1, and $10 M_{\odot}$, respectively, in all the panels.

expected, the cold gas mass is larger if $\dot{M}_{\mathrm{in}, 0}$ is larger, but the logarithmic amplitude of the cold gas mass does not depend on $\dot{M}_{\text {in, } 0}$, since we fix the time-scales of AGN feedback and mass transport.

\subsection{Mass transport time-scale in AGN feedback}

The mass transport time-scale of AGN feedback, $\tau_{\text {tr }}$, regulates the mass loss rate of the cold gas when AGN feedback is on (equation 6). In Fig. 3, we show the time evolution of the quantities of interest for $\tau_{\mathrm{tr}}=10^{7}$ (fiducial), $3 \times 10^{6}$, and $3 \times 10^{7}$ yr.

The increase and decrease of the dust-to-gas ratio in the hot gas is sharper if $\tau_{\text {tr }}$ is shorter just because the effect of mass transport from the cold to hot gas occurs on a shorter time-scale. The amplitudes of all the quantities shown in Fig. 3 are larger for shorter $\tau_{\text {tr }}$ because larger mass is exchanged between the cold and hot phases. In particular, the amplitude of the cold gas mass is sensitive to $\tau_{\text {tr }}$. Accordingly, the dust-to-gas ratio in the cold gas drops more for shorter $\tau_{\text {tr }}$ because the effect of dilution by the inflow is more significant. In contrast, the dust-to-gas ratio in the hot gas drops to the value insensitive to $\tau_{\text {tr }}$ when the AGN feedback is off (i.e., when there is no supply of dust from the cold gas) because of sputtering. The level of the lowest $\mathcal{D}_{\mathrm{h}}$ is determined by the balance between dust formation by AGB stars and dust destruction by sputtering.

\subsection{Duration of AGN feedback}

The duration of AGN feedback relative to the AGN duty cycle is parametrized by $f_{\text {ret }}$, so that an episode of AGN feedback 


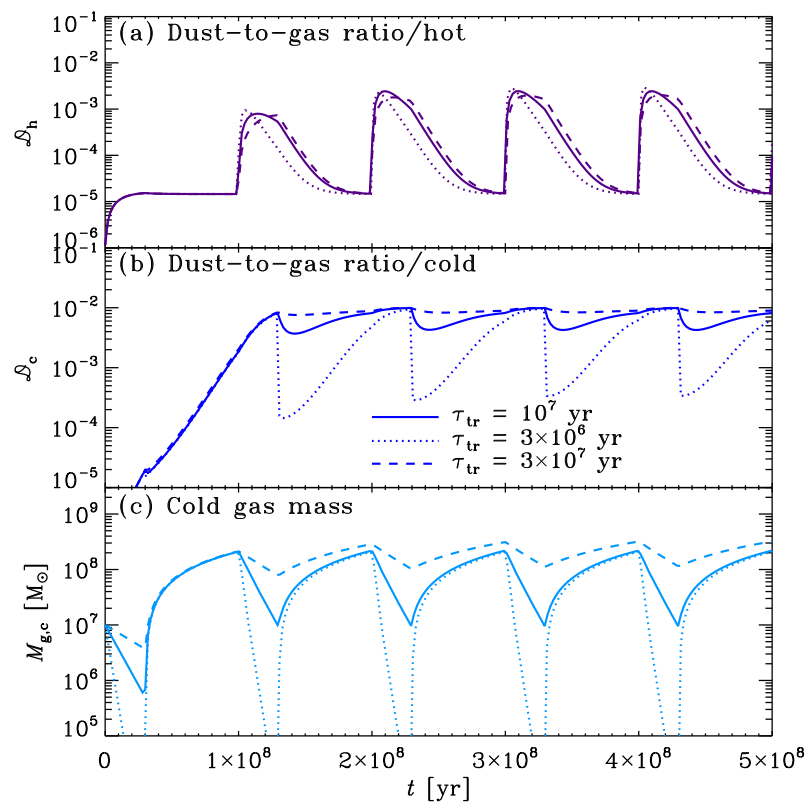

Figure 3: Same as Fig. 2 but for various mass transport times $\left(\tau_{\mathrm{tr}}\right)$. The solid, dotted, and dashed lines show the results for $\tau_{\mathrm{tr}}=10^{7}$ (fiducial), $3 \times 10^{6}$, and $3 \times 10^{7} M_{\odot}$, respectively, in all the panels.

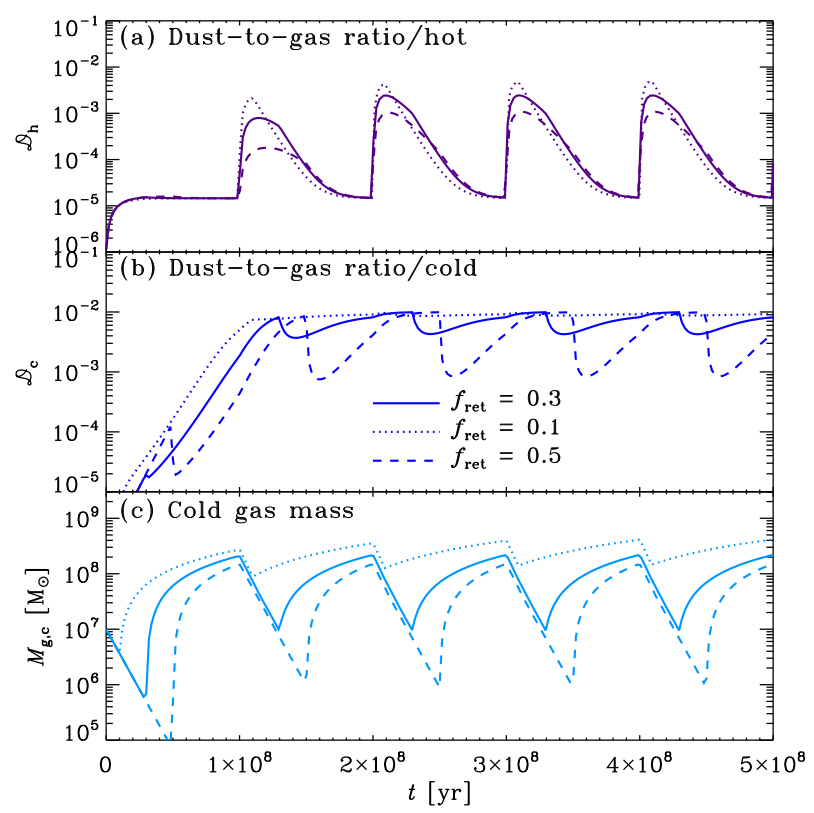

Figure 4: Same as Fig. 2 but for various ratios of the AGN feedback duration to the AGN feedback duty cycle time $\left(f_{\text {ret }}\right)$. The solid, dotted, and dashed lines show the results for $f_{\text {ret }}=0.3$ (fiducial), 0.1 , and 0.5 , respectively, in all the panels.

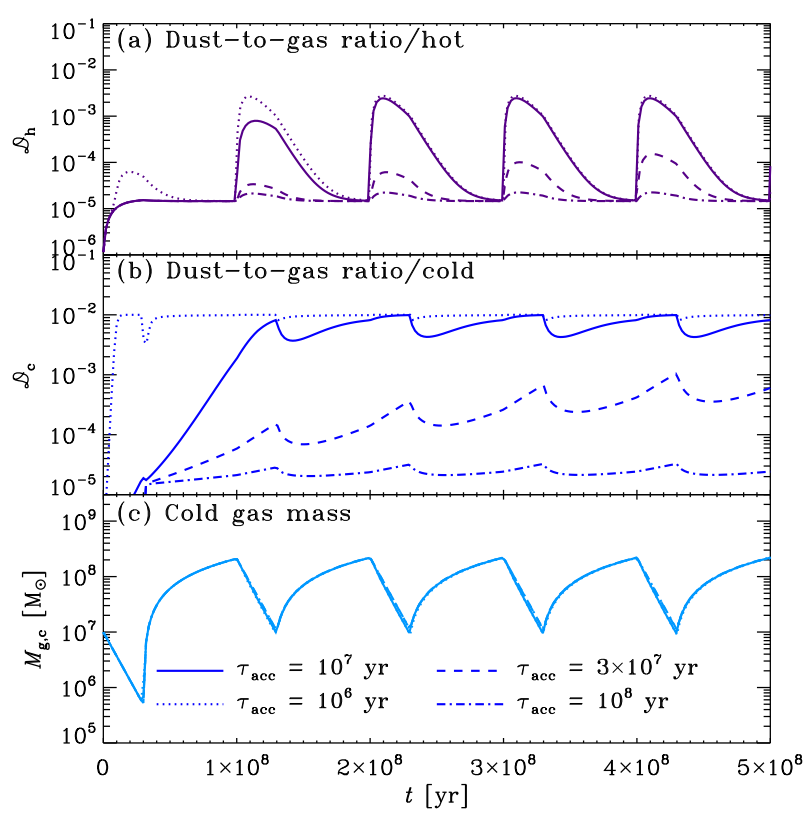

Figure 5: Same as Fig.2 2 but for various time-scales of dust growth by accretion $\left(\tau_{\text {acc }}\right)$. The solid, dotted, dashed, and dot-dashed lines show the results for $\tau_{\text {acc }}=10^{7}$ (fiducial), $10^{6}, 3 \times 10^{7}$ and $10^{8} \mathrm{yr}$, respectively, in all the panels.

lasts for $f_{\text {ret }} \tau_{\mathrm{AGN}}$. In Fig. 4, we show the time evolution of the quantities of interest for $f_{\text {ret }}=0.3$ (fiducial), 0.1, and 0.5.

Comparing Figs. 3 and 4 , we find that the duration of AGN feedback has an effect similar to the mass transport time in terms of the amplitudes: a larger $f_{\text {ret }}$ makes the amplitudes of the cold gas mass and the dust-to-gas ratio in the cold gas larger. The cold gas naturally decreases more if AGN feedback lasts longer, and the subsequent dilution of dust abundance by inflow becomes more significant. The dust-to-gas ratio in the hot gas has a larger amplitude for a smaller $f_{\text {ret }}$ since the cold gas mass is larger (i.e., the dust mass supplied from the cold gas is larger).

\subsection{Dust growth time-scale}

The accretion time-scale $\tau_{\text {acc }}$ regulates the dust growth in the cold gas. In Fig. 5, we show the time evolution of the quantities of interest for $\tau_{\text {acc }}=10^{7}$ (fiducial), $10^{6}, 3 \times 10^{7}$, and $10^{8} \mathrm{yr}$.

Naturally, the cold gas mass, which is not related to dust, is not affected by $\tau_{\text {acc }}$. As expected, the dust-to-gas ratio in the cold gas becomes higher for shorter $\tau_{\text {acc }}$. Moreover, if $\tau_{\text {acc }}$ is as long as $\tau_{\mathrm{AGN}}$, the overall level of dust-to-gas ratio is suppressed because dust does not grow sufficiently within an AGN feedback cycle. The dust-to-gas ratio in the hot gas is also affected, since the major source of dust in the hot gas is the dust grown in the cold gas. We observe a secular increase of $\mathcal{D}_{\mathrm{c}}$ for $\tau_{\text {acc }}=3 \times 10^{7} \mathrm{yr}$. This indicates that, if $\tau_{\text {acc }}$ is smaller than $\tau_{\mathrm{AGN}}$, the dust-to-gas ratio in the cold gas tends to increase unless it reaches the saturation limit $\left(\mathcal{D}_{\text {sat }}\right)$. Therefore, we observe a bifurcation for the value of $\mathcal{D}_{\mathrm{c}}$ : if $\tau_{\mathrm{acc}}<\tau_{\mathrm{AGN}}$, the dust-to-gas ratio increases while if $\tau_{\mathrm{acc}}>\tau_{\mathrm{AGN}}$, the dust-to-gas ratio is suppressed and $\mathcal{D}_{\mathrm{c}} \sim \mathcal{D}_{\mathrm{h}}$. In the latter case, dust growth has little 


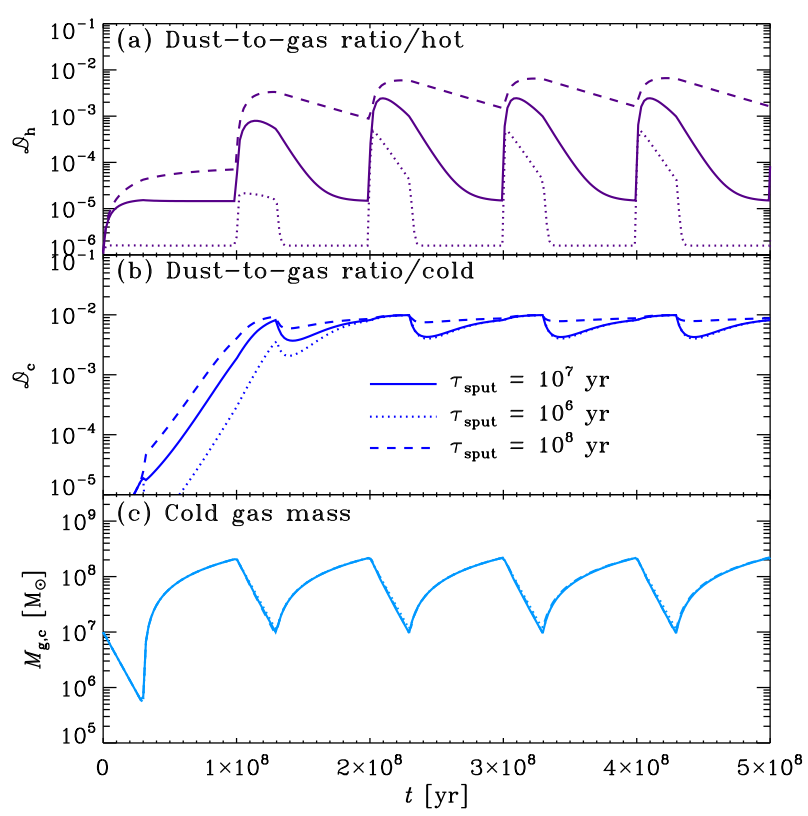

Figure 6: Same as Fig. 2 but for various time-scales of dust destruction by sputtering $\left(\tau_{\text {sput }}\right)$. The solid, dotted, and dashed lines show the results for $\tau_{\text {sput }}=10^{7}$ (fiducial), $10^{6}$, and $10^{8} \mathrm{yr}$, respectively, in all the panels.

influence on the dust budget and the dust-to-gas ratio is simply determined by the balance between dust supply from AGB stars and dust destruction in the hot gas.

\subsection{Dust destruction time-scale}

Dust in the hot gas is destroyed by sputtering. The destruction time-scale is regulated by $\tau_{\text {sput }}$. In Fig. 6, we show the time evolution of the relevant quantities for $\tau_{\text {sput }}=10^{7}$ (fiducial), $10^{6}$, and $10^{8} \mathrm{yr}$.

Naturally, the cold gas mass, which is not related to dust, is not affected by $\tau_{\text {sput }}$. As expected, the dust-to-gas ratio in the hot gas is largely affected by the sputtering time-scale, with longer $\tau_{\text {sput }}$ predicting higher $\mathcal{D}_{\mathrm{h}}$. The dust-to-gas ratio in the hot gas is roughly proportional to $\tau_{\text {sput }}$ (i.e., inversely proportional to the dust destruction efficiency). The dust-to-gas ratio in the cold gas is also kept high for $\tau_{\text {sput }}=10^{8} \mathrm{yr}$ : if $\mathcal{D}_{\mathrm{h}}$ remains as high as $\mathcal{D}_{c}$, the dust-to-gas ratio in the cold gas is kept high because the dust-to-gas ratio in the infalling gas is high (i.e., the dust abundance in the cold gas is not diluted by the infall). This effect is significant only if $\tau_{\text {sput }} \gtrsim \tau_{\mathrm{AGN}}$.

\subsection{Stellar mass}

The total stellar mass affects the dust evolution through dust production by AGB stars. In Fig.77, we show the time evolution of the relevant quantities for $M_{*}=10^{10.5}$ (fiducial), $10^{10}$, and $10^{11} M_{\odot}$.

The stellar mass does not affect the cold gas mass in our model since it only contributes to the dust production. Because the dust supply from AGB stars to the hot gas is proportional to the stellar mass, the lowest level of dust-to-gas ratio which

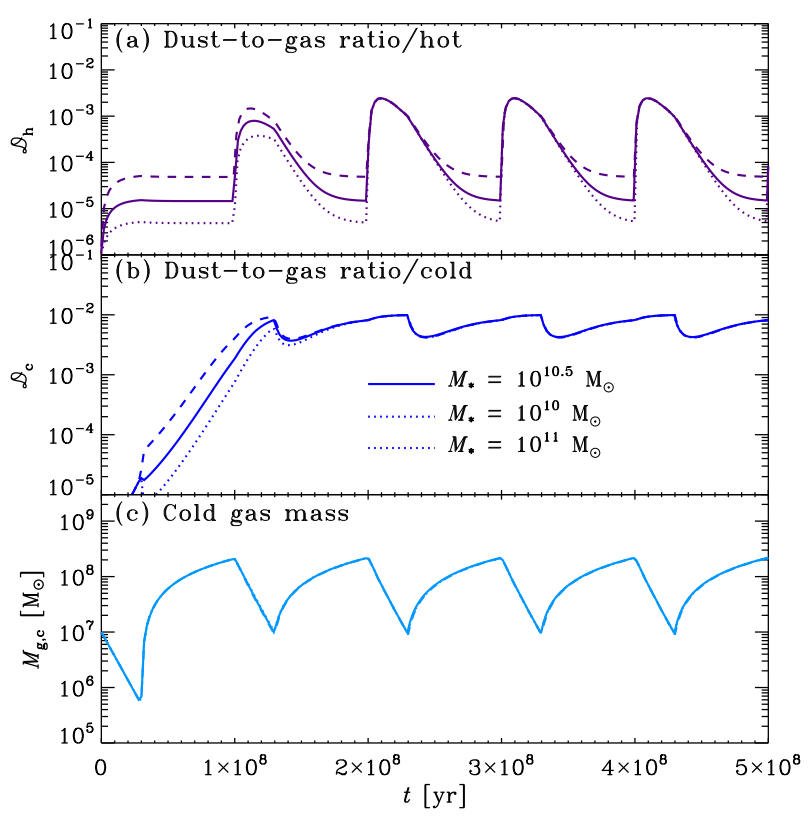

Figure 7: Same as Fig. 2 but for various stellar masses $\left(M_{*}\right)$. The solid, dotted, and dashed lines show the results for $M_{*}=10^{10.5}$ (fiducial), $10^{10}$, and $10^{11} M_{\odot}$, respectively, in all the panels.

is determined by the balance between dust supply and dust destruction is proportional to the stellar mass. The maximum level of the dust-to-gas ratio in the hot gas is insensitive to the stellar mass, because it is determined by the dust injection from the cold gas. The dust-to-gas ratio in the cold gas is not sensitive to the stellar mass as long as dust growth is efficient enough.

\section{Discussion}

In this section, we mainly compare the theoretical calculations with observational data. Here we utilize the observational data for dust and gas in elliptical galaxies. Because of the general deficiency of gas and dust in elliptical galaxies, data is limited, while the stellar mass is relatively easy to be determined in these systems using optical photometric observations. Thus, we also adopt the stellar mass and utilize it (mainly for normalization).

\subsection{Sample}

One of the newest systematic dust observations in nearby elliptical galaxies is carried out by Herschel. We adopt the sample from Smith et al. (2012) and di Serego Alighieri et al. (2013). The sample galaxies adopted are listed in Table 2 We selected galaxies with a morphological type of elliptical galaxies (E) and excluded objects with stellar mass with $<10^{9.5} M_{\odot}$. NGC 4374 is common for both samples. The stellar mass is taken from Cortese et al. (2012). The gas masses are traced by $\mathrm{H}_{\mathrm{I}}$ and $\mathrm{H}_{2}$ (CO); therefore, the observed gas masses are compared with the cold gas mass in our models. We only compare the observed dust mass with the dust mass in the cold gas in our models, because the observational sensitivity to the diffuse component 
in the hot gas depends on the spatial extension, which is not known. In any case, since the dust mass in the cold gas is much larger than that in the hot gas in most of the time, adding the dust mass in the hot gas does not significantly change the comparison below.

\subsection{Cold gas mass}

The variation of the cold gas mass in our models in Section 3 is broadly in the range of the observed gas masses shown in Table 2 ( $\sim 10^{9} M_{\odot}$ down to $\sim 10^{7} M_{\odot}$ or less $)$. Therefore, we confirm that the choices of parameter values in Section 2 are reasonable as far as the cold gas mass is concerned. The largest cold gas mass is realized if we adopt the largest inflow rate $\left(\dot{M}_{\text {in, } 0}=10 M_{\odot} \mathrm{yr}^{-1}\right.$; Fig. 2). Therefore, we confirm that the maximum inflow rate by the accretion of the cooled gas is $\lesssim$ $10 M_{\odot} \mathrm{yr}^{-1}$. The amplitude of the cold gas mass is, on the other hand, determined by the time-scale of gas transport in AGN feedback (Fig. 3) and the duration of AGN feedback (Fig. 4). If the variation of the gas mass in the sample is interpreted as due to time evolution, $\tau_{\text {tr }} \gtrsim 3 \times 10^{7}$ yr or $f_{\text {ret }} \lesssim 0.1$ is not favored because the variation of the cold gas is smaller than an order of magnitude. The non-detection of $\mathrm{H}_{\mathrm{I}}$ and $\mathrm{H}_{2}$ gas for a large part of the sample galaxies is consistent with even the most extreme case of $\tau_{\text {tr }}=3 \times 10^{6} \mathrm{yr}$ or $f_{\text {ret }}=0.5$. This means that it is difficult to constrain the lower bound for $\tau_{\text {tr }}$ and the upper bound for $f_{\text {ret }}$.

\subsection{Dust mass}

The dust mass in the cold gas varies between $\sim 10^{5}$ and $\sim$ $10^{6} M_{\odot}$ in the fiducial model (Fig. 1). The maximum of the dust mass is achieved when the cold gas mass becomes the largest (i.e., just before AGN feedback). In this phase, the dust-togas ratio is almost saturated if $\tau_{\text {acc }}$ is significantly shorter than $\tau_{\mathrm{AGN}}$. If we adopt the maximum value of the dust-to-gas ratio $\left(\mathcal{D}_{\text {sat }}=0.01\right)$, we obtain the maximum dust mass in the case of the largest cold gas mass $\left(10^{9} M_{\odot}\right.$; see above $)$ as $\sim 10^{7} M_{\odot}$. The observed dust mass is indeed smaller than this value. Because the logarithmic variation of the dust-to-gas ratio is smaller than that of the cold gas mass, the time evolution of the dust mass is dominated by the change of the cold gas mass in our models.

\subsection{Dust-to-stellar mass ratio}

To cancel out the size effects of elliptical galaxies, it is useful to normalize the dust mass to an indicator of total mass scale. Here we use the stellar mass for the normalization; that is, we use the dust-to-stellar mass ratio for the indicator of the richness of dust. As an indicator of AGN feedback, we also show the ratio of the cold gas mass to the stellar mass, and examine the relation between $M_{\mathrm{d}, \mathrm{c}} / M_{*}$ and $M_{\mathrm{g}, \mathrm{c}} / M_{*}$. For the data points, we exclude the objects without any constraint on the $\mathrm{H}$ i mass, since it is impossible to constrain the gas mass in such a case. If $\mathrm{H}_{\mathrm{I}}$ is detected, we sum the $\mathrm{H}_{\mathrm{I}}$ mass and the $\mathrm{H}_{2}$ mass to obtain the total cold gas mass (if $\mathrm{H}_{2}$ is not detected, we add the upper limit of $\mathrm{H}_{2}$ gas mass to the $\mathrm{H}_{\mathrm{I}}$ gas mass).

In Fig. 8, we show the relation between $M_{\mathrm{d}, \mathrm{c}} / M_{*}$ and $M_{\mathrm{g}, \mathrm{c}} / M_{*}$. We only show the dependence on the parameters which affect the relative abundance of dust to gas (i.e., dustto-gas ratio). Those parameters are $\tau_{\text {tr }}, f_{\text {ret }}, \tau_{\text {acc }}$ and $\tau_{\text {sput }}$. The same ranges for the parameters as above (i.e., the ranges in $\mathrm{Ta}-$ ble 1) are investigated. We only plot the relation at $t>2 \times 10^{8} \mathrm{yr}$, when the trajectory almost converges to a limit cycle (by "forgetting the initial condition") except for the case with $\tau_{\text {acc }}=$ $3 \times 10^{7} \mathrm{yr}$ in Panel (c). In this case, there is a secular drift toward high dust abundance as shown in Fig. 5

Overall, we observe in Fig. 8 that the variation is dominated by the change of the gas mass because the variation is mostly in the diagonal direction on the diagram. If the gas mass changes, both $M_{\mathrm{d}, \mathrm{c}} / M_{*}$ and $M_{\mathrm{g}, \mathrm{c}} / M_{*}$ moves on the diagonal line under a constant $\mathcal{D}_{c}$. In other words, the variation of the dust-to-gas ratio can be seen in off-diagonal behavior. Indeed, such offdiagonal behavior is seen clearly for a short $\tau_{\text {tr }}$ in Fig. 8a and a large $f_{\text {ret }}$ in Fig. 8b. In both cases, the cold gas mass is lost drastically in the epoch of AGN feedback, so that the dilution of dust abundance by the inflow of dust-poor gas has a large impact on the dust-to-gas ratio in the cold gas (Sections 3.2 and 3.4).

We observe in Fig. 8 that a single model does not cover an area wide enough to explain all the data points. In particular, the data points at high $M_{\mathrm{g}, \mathrm{c}} / M_{*}$ and low $M_{\mathrm{d}, \mathrm{c}} / M_{*}$ are difficult to be reproduced. As shown above, the dilution effect appears just after the epoch of AGN feedback, when the cold gas mass is low. Therefore, it is difficult to reproduce the low dust content at the high gas mass. In our scenario, the only way to reproduce such a dust-poor and gas-rich data point is to change the efficiency of accretion as shown in Fig. 8k. The same effect is also realized by lowering $\mathcal{D}_{\text {sat }}$ (equation 9 . Therefore, the large dispersion of dust mass at high gas mass can be interpreted as various dust growth efficiencies. A variety in dust growth efficiency could be caused by a variety in dense gas fraction, cold gas density, metallicity, or grain size distribution (Section 2.3).

Some studies argue that the dust-to-stellar mass ratio changes as the system is enriched with dust (e.g., Rémy-Ruyer et al., 2015). Dust enrichment is indeed the most important factor of changing the dust-to-stellar mass ratio in gas-rich star-forming galaxies, since dust production by stars and dust growth in the cold dense ISM are actively occurring. In this paper, we have shown that, in elliptical galaxies, the dust-to-stellar mass ratio is still varied by dust growth, although it is driven by a cycle of AGN feedback, not by chemical enrichment. Therefore, our results imply that, if AGN feedback affects the galaxy evolution, we also need to consider the variation of dust abundance by AGN feedback.

\subsection{Dust-to-gas ratio}

As shown above, the behavior on the $M_{\mathrm{d}, \mathrm{c}} / M_{*}-M_{\mathrm{g}, \mathrm{c}} / M_{*} \mathrm{di}-$ agram is dominated by the overall oscillation of the cold gas mass. To examine the effect of dust abundance variation more clearly, it is useful to consider a quantity normalized to the gas mass. In this way, we can cancel the effect of oscillatory behavior of the cold gas mass, and concentrate on the variation of dust abundance. We still use the gas-to-stellar mass ratio, $M_{\mathrm{g}, \mathrm{c}} / M_{*}$, for the horizontal axis to show the cycle of AGN feedback. Thus, we consider the $\mathcal{D}_{\mathrm{c}}-M_{\mathrm{g}, \mathrm{c}} / M_{*}$ diagram here. 
Table 2: Elliptical galaxy sample adopted from Smith et al. (2012) and di Serego Alighieri et al. (2013).

\begin{tabular}{|c|c|c|c|c|c|c|c|}
\hline Name & Other name & $\begin{array}{c}\log M_{*} \\
\left(M_{\odot}\right) \\
\end{array}$ & $\begin{array}{c}\log M_{\text {dust }} \\
\left(M_{\odot}\right)\end{array}$ & $\begin{array}{c}\log M_{\mathrm{HI}} \\
\left(M_{\odot}\right)\end{array}$ & $\begin{array}{c}\log M_{\mathrm{H}_{2}} \\
\left(M_{\odot}\right) \\
\end{array}$ & $\log \mathcal{D}$ & Ref. $^{a}$ \\
\hline VCC 763 & NGC 4374, M84 & 11.18 & $5.05^{b}$ & 8.96 & $<7.23$ & $-3.91^{c}$ & $1,2,3$ \\
\hline HRS 150 & NGC 4406, M86 & 11.22 & 6.63 & 7.95 & $<7.4$ & $-1.43^{c}$ & 1,3 \\
\hline HRS 186 & NGC 4494 & 10.88 & 5.08 & 8.26 & $<7.35$ & $-3.23^{c}$ & 1,3 \\
\hline HRS 241 & NGC 4636 & 10.98 & 5.06 & 9.0 & $<7.02$ & $-3.94^{c}$ & 1,3 \\
\hline VCC 345 & NGC 4261 & 11.32 & 5.81 & $<8.45$ & $<7.70$ & $>-2.71^{d}$ & 2,3 \\
\hline VCC 881C & NGC 4406 & 11.22 & 5.47 & 7.95 & - & $-2.48^{e}$ & 2,3 \\
\hline VCC 1226 & NGC 4472, M49 & 11.39 & 5.49 & $<7.90$ & $<7.26$ & $>-2.50^{d}$ & 2,3 \\
\hline VCC 1619 & NGC 4550 & 10.03 & 5.41 & $<7.90$ & 7.20 & $>-2.57^{d}$ & 2,3 \\
\hline HRS 3 & NGC 3226 & 10.21 & 5.96 & - & $<7.13$ & - & 1,3 \\
\hline HRS 43 & NGC 3608 & 10.27 & $<4.88$ & — & $<7.28$ & - & 1,3 \\
\hline HRS 49 & NGC 3640 & 10.70 & $<5.20$ & - & $<7.25$ & - & 1,3 \\
\hline HRS 125 & NGC 4339 & 10.30 & $<5.17$ & $<7.84$ & $<7.2$ & - & 1,3 \\
\hline HRS 135 & NGC 4365 & 11.48 & $<6.17$ & $<8.18$ & $<7.62$ & 一 & 1,3 \\
\hline HRS 179 & NGC 4473 & 10.71 & $<5.19$ & $<7.92$ & $<7.16$ & - & 1,3 \\
\hline HRS 181 & NGC 4478 & 10.09 & $<4.85$ & - & $<7.31$ & - & 1,3 \\
\hline HRS 211 & NGC 4552, M89 & 10.80 & $<5.67$ & $<7.92$ & $<7.36$ & - & 1,3 \\
\hline HRS 214 & NGC 4564 & 10.25 & $<5.30$ & $<7.79$ & $<7.33$ & - & 1,3 \\
\hline HRS 218 & NGC 4570 & 10.48 & $<5.67$ & $<7.31$ & $<7.47$ & - & 1,3 \\
\hline HRS 236 & NGC 4621, M59 & 10.99 & $<5.76$ & $<7.92$ & $<7.24$ & - & 1,3 \\
\hline HRS 245 & NGC 4649, M60 & 11.34 & $<5.40$ & $<7.92$ & $<7.59$ & 一 & 1,3 \\
\hline HRS 248 & NGC 4660 & 10.05 & $<4.85$ & $<7.92$ & $<7.30$ & - & 1,3 \\
\hline HRS 258 & NGC 4697 & 11.10 & 5.46 & - & $<7.25$ & - & 1,3 \\
\hline HRS 312 & NGC 5576 & 10.60 & $<5.51$ & - & $<7.46$ & - & 1,3 \\
\hline HRS 316 & NGC 5638 & 10.52 & $<5.23$ & - & $<7.54$ & - & 1,3 \\
\hline VCC 1316 & NGC 4486, M87 & 11.24 & 5.34 & 一 & $<7.18$ & - & 2,3 \\
\hline VCC 1327 & NGC 4486A & 10.05 & 4.92 & 一 & - & 一 & 2,3 \\
\hline
\end{tabular}

${ }^{a}$ References: 1) Smith et al. (2012); 2) di Serego Alighieri et al. (2013); 3) Cortese et al. (2012).

${ }^{b}$ Adopted from Ref. 1. Ref. 2 gives 5.30 with the same mass absorption coefficient.

${ }^{c}$ The upper limit of $\mathrm{H}_{2}$ mass is used, but this does not affect the estimated dust-to-gas ratio since the gas mass is dominated by Hi gas mass.

${ }^{d}$ We use the upper limits of gas mass to derive the lower limits of dust-to-gas ratio.

${ }^{e}$ We neglect the $\mathrm{H}_{2}$ mass for the gas mass. 

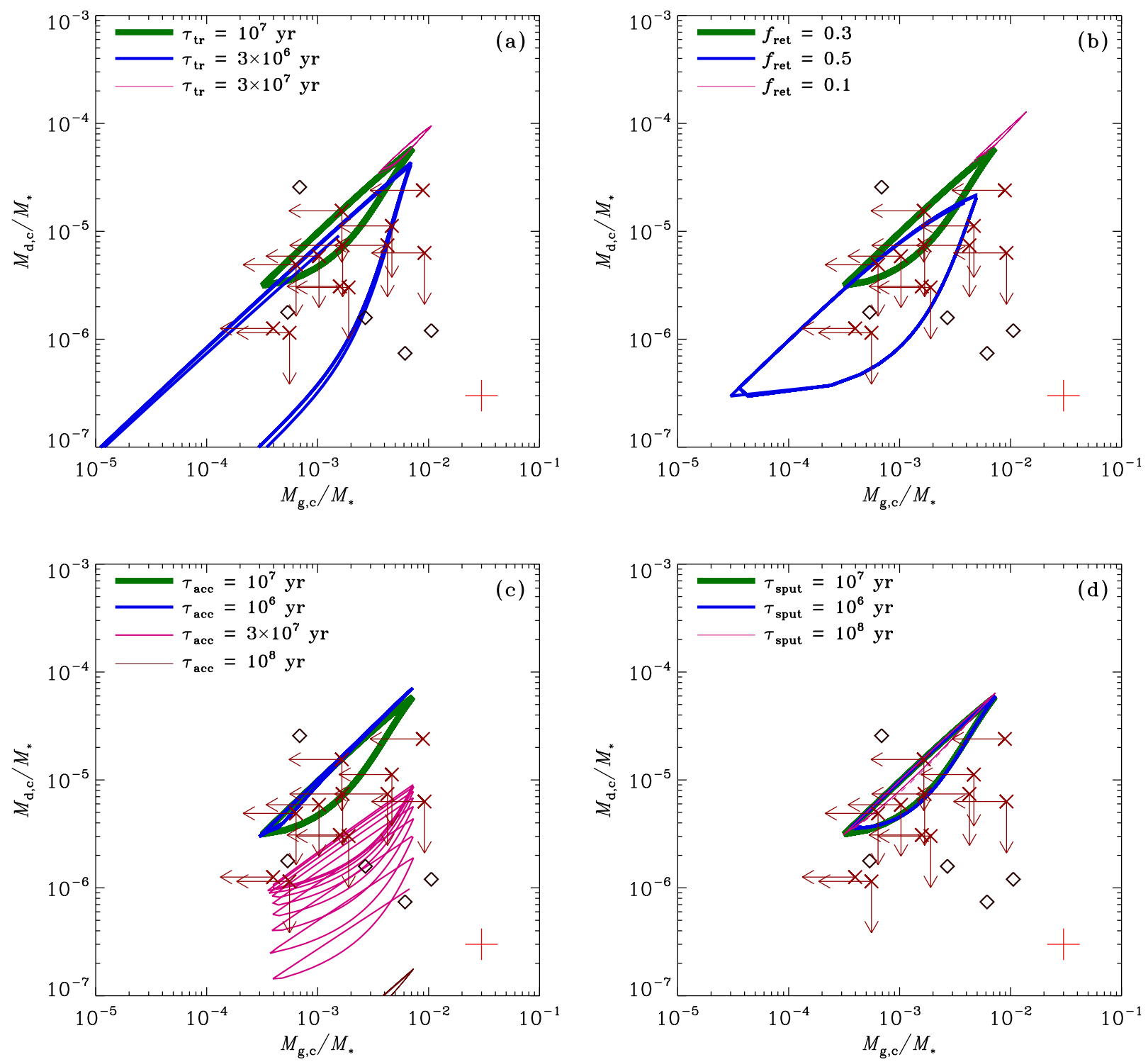

Figure 8: Relation between dust-to-stellar mass ratio $\left(M_{\mathrm{d}, \mathrm{c}} / M_{*}\right)$ and gas-to-stellar mass ratio $\left(M_{\mathrm{g}, \mathrm{c}} / M_{*}\right)$. (a) Dependence on $\tau_{\mathrm{tr}}$. The thick (green), medium (blue) and thin (red) lines represent the trajectory for $\tau_{\text {tr }}=10^{7}$ (fiducial), $3 \times 10^{6}$, and $3 \times 10^{7} \mathrm{yr}$, respectively. (b) Dependence on $f_{\text {ret }}$. The thick (green), medium (blue), and thin (red) lines show the trajectory for $f_{\text {ret }}=0.3$ (fiducial), 0.5 , and 0.1 , respectively. (c) Dependence on $\tau_{\text {acc. }}$ The thick (green), medium thick (blue), medium thin (red), and thin (brown) lines represent the trajectory for $\tau_{\text {acc }}=10^{7}$ (fiducial) $10^{6}, 3 \times 10^{7}$, and $10^{8} \mathrm{yr}$, respectively. (d) Dependence on $\tau_{\text {sput }}$. The thick (green), medium (blue), and thin (red) lines show the trajectory for $\tau_{\text {sput }}=10^{7}$ (fiducial), $10^{6}$, and $10^{8} \mathrm{yr}$, respectively. These three lines are almost overlapped. We also show observational data points in all the panels listed in Table 2 The diamonds show the galaxies for which both dust mass and gas mass are detected, while the crosses with arrows present the data points for which either dust mass or gas mass is not detected (only an upper limit is obtained). The cross on the right lower corner shows the typical error for the observational data points. 
The same observational sample as in Section 4.4 is chosen for comparison but we excluded galaxies without detection of dust emission.

In Fig. 9, we show the relation between $\mathcal{D}_{\mathrm{c}}$ and $M_{\mathrm{g}, \mathrm{c}} / M_{*}$. We only plot the relation at $t>2 \times 10^{8} \mathrm{yr}$ as also done in Fig. 8 . We examine the variations of the same parameters as in Section 4.4. The horizontal axis shows the "phase" of AGN feedback with high and low phases of $M_{\mathrm{g}, \mathrm{c}} / M_{*}$ showing the timings when AGN feedback is turned on and off, respectively. The upper limit of $\mathcal{D}_{\mathrm{c}}$ is determined by the saturation of dust growth $\left(\mathcal{D}_{\text {sat }}\right)$. After AGN feedback, $\mathcal{D}_{\mathrm{c}}$ becomes low by the dilution as a result of the inflow of dust-poor gas. As the cold gas mass increases, dust growth raises the dust-to-gas ratio.

As concluded in Section 4.4, it is difficult to reproduce the dust-poor objects at high gas mass, and the only way to reproduce them in our model is to assume inefficient dust growth. However, it should be emphasized that our model is capable of reproducing an order of magnitude variation in dust-to-gas ratio if AGN feedback efficiently evaporates the cold gas into the hot gas $\left(\tau_{\text {tr }}=3 \times 10^{6} \mathrm{yr}\right.$ in Fig. $9 \mathrm{a}$ and $f_{\text {ret }}=0.5$ in Fig. $\left.9 \mathrm{~b}\right)$.

\section{Conclusion}

In this paper, we modeled the evolution of dust in elliptical galaxies and investigated the dust formation and evolution in the cold and hot gas components. We also considered the mass exchange between the two gas components by including AGN feedback, which heats the cold gas and converts it to hot gas, and also by considering cooling of the hot gas, which is accreted on to the cold gas. We considered a new dust formation path through dust growth by the accretion of gas-phase metals in addition to dust formation by AGB stars. Thus, the injection of the cold gas into the hot gas by AGN feedback acts as a dust supplying mechanism to the hot gas. We also included sputtering in the hot gas following previous papers. Since sputtering is efficient enough to make the dust-to-gas ratio in the hot gas much lower than that in the cold gas, the mass inflow to the cold gas dilutes the dust abundance there.

We surveyed reasonable ranges of the relevant parameters in the model and clarified how each process affects the evolution of dust and gas in elliptical galaxies. (i) The mass inflow rate into the cold gas naturally affects the cold gas mass, although it has a minor influence on the dust-to-gas ratio in the cold gas. Therefore, the role of mass inflow is to characterize the total mass scale of the cold gas and dust. (ii) The mass transport time-scale from the cold gas to the hot gas in AGN feedback affects the amplitudes of the cold gas mass and the dust-to-gas ratio in the cold gas. If this time-scale is shorter, the cold gas loses a larger fraction of its mass in AGN feedback. As a result, the subsequent inflow of dust-poor gas dilutes the dust abundance in the cold gas more. Therefore, the variation of dust-togas ratio in the cold gas is larger for a shorter mass transport time-scale. (iii) The duration of AGN feedback also regulates the cold gas lost in an AGN feedback cycle. Therefore, its effect is similar to that of the mass transport time-scale, and the amplitude of the dust-to-gas ratio in the cold gas is larger for a longer duration of AGN feedback. (iv) The dust growth time-scale in the cold gas has the largest impact on the dust-to-gas ratio there. It affects the absolute level of dust-to-gas ratio rather than the amplitude of dust-to-gas ratio. The low dust-to-gas ratios in some relatively gas-rich elliptical galaxies could be most naturally explained by a large variation in dust growth time-scale among the sample. Such a variation can be a consequence of a variety in dense gas fraction, grain size distribution, metallicity, or gas density. (v) As long as the sputtering time-scale is significantly shorter than the AGN feedback cycle, dust in the hot gas is destroyed efficiently so that the infall from the hot to the cold gas phase always works as dilution of the dust abundance in the cold gas. However, if the sputtering time-scale is comparable or longer than the AGN feedback cycle, the dilution of the dust abundance in the cold gas does not occur efficiently (i.e., the dust-to-gas ratio in the hot gas remains high), so that the dust-to-gas ratio in the cold gas does not drop after the inflow. (vi) Because the major source of dust is dust growth in the cold gas, dust production by AGB stars does not affect the evolution of the dust-to-gas ratio in the cold gas.

By comparing with observational data of nearby elliptical galaxies, we showed that the variety in gas mass is nicely explained by our models and that the variety in the dust-to-gas ratio can also be reproduced to some extent. However, the full range of the observed dust-to-gas ratio is difficult to be reproduced unless we vary the dust growth time-scale (or dust growth efficiency). In our framework, the low dust-to-gas ratios in relatively gas-rich objects are only reproduced with inefficient dust growth (or long dust growth time-scales). Therefore, we conclude that dust growth can play a central role in creating the variation in dust-to-gas ratio through the AGN feedback cycle and through the variation in dust growth efficiency.

\section{Acknowledgment}

We are grateful to T. Kokusho and H. Kaneda for their useful comments and discussions, and to the anonymous referees for their careful reviews of the paper. $\mathrm{HH}$ is supported by the Ministry of Science and Technology (MoST) grant 102-2119M-001-006-MY3. TN is supported in part by the JSPS Grantin-Aid for Scientific Research (26400223).

\section{References}

Aoyama, S., Hou, K.-C., Shimizu, I., Hirashita, H., Todoroki, K., Choi, J.-H., Nagamine, K., 2017. Galaxy simulation with dust formation and destruction. Mon. Not. R. Astron. Soc. 466, 105-121.

Asano, R. S., Takeuchi, T. T., Hirashita, H., Inoue, A. K., 2013. Dust formation history of galaxies: A critical role of metallicity for the dust mass growth by accreting materials in the interstellar medium. Earth, Planets, and Space 65, 213-222.

Booth, C. M., Schaye, J., 2009. Cosmological simulations of the growth of supermassive black holes and feedback from active galactic nuclei: method and tests. Mon. Not. R. Astron. Soc. 398, 53-74.

Buote, D. A., Lewis, A. D., Brighenti, F., Mathews, W. G., 2003. XMMNewton and Chandra Observations of the Galaxy Group NGC 5044. I. Evidence for Limited Multiphase Hot Gas. Astrophys. J. 594, 741-757.

Ciotti, L., Ostriker, J. P., 2001. Cooling Flows and Quasars. II. Detailed Models of Feedback-modulated Accretion Flows. Astrophys. J. 551, 131-152. 

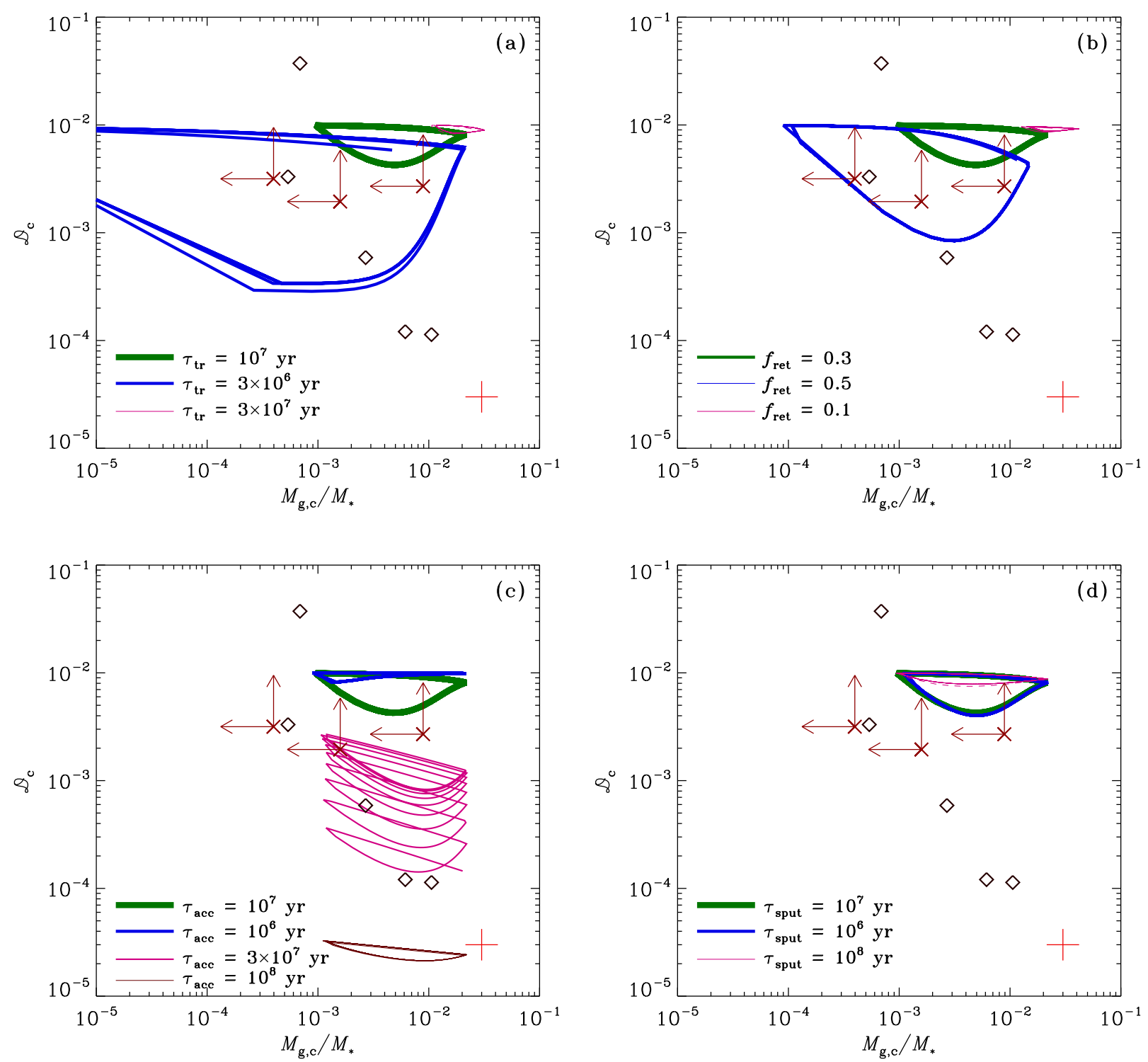

Figure 9: Same as Fig. 8 but showing the relation between dust-to-gas ratio $\left(\mathcal{D}_{\mathrm{c}}=M_{\mathrm{d}, \mathrm{c}} / M_{\mathrm{g}, \mathrm{c}}\right)$ and gas-to-stellar mass ratio $\left(M_{\mathrm{g}, \mathrm{c}} / M_{*}\right)$. We also show observational data points listed in Table 2 (but only the objects with dust detection are shown): The diamonds show the galaxies for which gas is detected, while the crosses with arrows present the data points without gas detection (only an upper limit is obtained for the gas mass). The cross on the right lower corner shows the typical error. 
Cortese, L., Boissier, S., Boselli, A., Bendo, G. J., Buat, V., Davies, J. I., Eales, S., Heinis, S., Isaak, K. G., Madden, S. C., 2012. The GALEX view of the Herschel Reference Survey. Ultraviolet structural properties of nearby galaxies. Astron. Astrophys. 544, A101.

Croton, D. J., Springel, V., White, S. D. M., De Lucia, G., Frenk, C. S., Gao, L., Jenkins, A., Kauffmann, G., Navarro, J. F., Yoshida, N., 2006. The many lives of active galactic nuclei: cooling flows, black holes and the luminosities and colours of galaxies. Mon. Not. R. Astron. Soc. 365, 11-28.

di Serego Alighieri, S., Bianchi, S., Pappalardo, C., Zibetti, S., Auld, R., Baes, M., Bendo, G., Corbelli, E., Davies, J. I., Davis, T., De Looze, I., Fritz, J., Gavazzi, G., Giovanardi, C., Grossi, M., Hunt, L. K., Magrini, L., Pierini, D., Xilouris, E. M., 2013. The Herschel Virgo Cluster Survey. XIII. Dust in early-type galaxies. Astron. Astrophys. 552, A8.

Dwek, E., 1987. The infrared diagnostic of a dusty plasma with applications to supernova remnants. Astrophys. J. 322, 812-821.

Dwek, E., 1998. The Evolution of the Elemental Abundances in the Gas and Dust Phases of the Galaxy. Astrophys. J. 501, 643.

Fabian, A. C., 1999. The obscured growth of massive black holes. Mon. Not. R. Astron. Soc. 308, L39-L43.

Fabian, A. C., 2012. Observational Evidence of Active Galactic Nuclei Feedback. Ann. Rev. Astron. Astrophys. 50, 455-489.

Fabian, A. C., Johnstone, R. M., Daines, S. J., 1994. The Effects of Dust in Cold Clouds Embedded in Cooling Flows. Mon. Not. R. Astron. Soc. 271, 737.

Ferrara, A., Viti, S., Ceccarelli, C., 2016. The problematic growth of dust in high-redshift galaxies. Mon. Not. R. Astron. Soc. 463, L112-L116.

Ferrari, F., Pastoriza, M. G., Macchetto, F., Caon, N., 1999. A survey of the ISM in early-type galaxies. II. The dust. Astron. Astrophys. Suppl. 136, 269-284.

Forbes, D. A., 1991. On the external origin for dust in elliptical galaxies. Mon. Not. R. Astron. Soc. 249, 779-787.

Fujita, Y., Okabe, N., Sato, K., Tamura, T., Matsushita, S., Hirashita, H., Nakamura, M., Matsushita, K., Nakazawa, K., Takizawa, M., 2013. Discovery of an Outstanding Disk in the cD Galaxy of the Hydra A Cluster. Publ. Astron. Soc. Japan 65, L15.

Gallazzi, A., Charlot, S., Brinchmann, J., White, S. D. M., Tremonti, C. A., 2005. The ages and metallicities of galaxies in the local universe. Mon. Not. R. Astron. Soc. 362, 41-58.

Goudfrooij, P., Hansen, L., Jorgensen, H. E., Norgaard-Nielsen, H. U., 1994. Interstellar matter in Shapley-Ames elliptical galaxies. II. The distribution of dust and ionized gas. Astron. Astrophys. Suppl. 105.

Hirashita, H., Jan. 1999. Global Law for the Dust-to-Gas Ratio of Spiral Galaxies. Astrophys. J. 510, L99-L102.

Hirashita, H., Kuo, T.-M., 2011. Effects of grain size distribution on the interstellar dust mass growth. Mon. Not. R. Astron. Soc. 416, 1340-1353.

Hirashita, H., Nozawa, T., Villaume, A., Srinivasan, S., 2015. Dust processing in elliptical galaxies. Mon. Not. R. Astron. Soc. 454, 1620-1633.

Hou, K.-C., Hirashita, H., Michałowski, M. J., 2016. Dust evolution processes constrained by extinction curves in nearby galaxies. Publ. Astron. Soc. Japan $68,94$.

Inoue, A. K., 2003. Evolution of Dust-to-Metal Ratio in Galaxies. Publ. Astron. Soc. Japan 55, 901-909.

Inoue, A. K., 2011. The origin of dust in galaxies revisited: the mechanism determining dust content. Earth, Planets, and Space 63, 1027-1039.

Kaneda, H., Ishihara, D., Onaka, T., Suzuki, T., Mori, T., Oyabu, S., Yamagishi, M., 2011. Properties of Dust and PAHs in the Hot Plasma of the Elliptical Galaxy NGC 4125 Revealed with AKARI and Spitzer Space Telescope. Publ. Astron. Soc. Japan 63, 601-615.

King, A., 2005. The AGN-Starburst Connection, Galactic Superwinds, and $\mathrm{M}_{B H^{-}} \sigma$. Astrophys. J. 635, L121-L123.

Knapp, G. R., Guhathakurta, P., Kim, D.-W., Jura, M. A., 1989. Interstellar matter in early-type galaxies. I - IRAS flux densities. Astrophys. J. Suppl. 70, 329-387.

Kuo, T.-M., Hirashita, H., Jul. 2012. Impact of grain size distributions on the dust enrichment in high-redshift quasars. Mon. Not. R. Astron. Soc. 424, L34-L38.

Kuo, T.-M., Hirashita, H., Zafar, T., Dec. 2013. Evolution of dust content in galaxies probed by gamma-ray burst afterglows. Mon. Not. R. Astron. Soc. 436, 1238-1244.

Lauer, T. R., Faber, S. M., Gebhardt, K., Richstone, D., Tremaine, S., Ajhar, E. A., Aller, M. C., Bender, R., Dressler, A., Filippenko, A. V., Green, R., Grillmair, C. J., Ho, L. C., Kormendy, J., Magorrian, J., Pinkney, J., Siopis,
C., 2005. The Centers of Early-Type Galaxies with Hubble Space Telescope. V. New WFPC2 Photometry. Astron. J. 129, 2138-2185.

Leeuw, L. L., Sansom, A. E., Robson, E. I., Haas, M., Kuno, N., 2004. Observations of Cold Dust in Nearby Elliptical Galaxies. Astrophys. J. 612, 837-847.

Mancini, M., Schneider, R., Graziani, L., Valiante, R., Dayal, P., Maio, U., Ciardi, B., Hunt, L. K., 2015. The dust mass in $z>6$ normal star-forming galaxies. Mon. Not. R. Astron. Soc. 451, L70-L74.

Mathews, W. G., Brighenti, F., 2003. Rapid Cooling of Dusty Gas in Elliptical Galaxies. Astrophys. J. 590, L5-L8.

Mathews, W. G., Temi, P., Brighenti, F., Amblard, A., 2013. Variations of Midand Far-infrared Luminosities among Early-type Galaxies: Relation to Stellar Metallicity and Cold Dust. Astrophys. J. 768, 28.

Mattsson, L., Andersen, A. C., 2012. On the dust abundance gradients in latetype galaxies - II. Analytical models as evidence for massive interstellar dust growth in SINGS galaxies. Mon. Not. R. Astron. Soc. 423, 38-48.

Mukherjee, D., Bicknell, G. V., Sutherland, R., Wagner, A., 2016. Relativistic jet feedback in high-redshift galaxies - I. Dynamics. Mon. Not. R. Astron. Soc. 461, 967-983.

Nozawa, T., Kozasa, T., Habe, A., 2006. Dust Destruction in the High-Velocity Shocks Driven by Supernovae in the Early Universe. Astrophys. J. 648, 435451

O’Sullivan, E., Forbes, D. A., Ponman, T. J., 2001. A catalogue and analysis of X-ray luminosities of early-type galaxies. Mon. Not. R. Astron. Soc. 328, $461-484$.

Patil, M. K., Pandey, S. K., Sahu, D. K., Kembhavi, A., 2007. Properties of dust in early-type galaxies. Astron. Astrophys. 461, 103-113.

Popping, G., Somerville, R. S., Galametz, M., 2016. The dust content of galaxies from $\mathrm{z}=0$ to $\mathrm{z}=9$. ArXiv e-prints.

Rémy-Ruyer, A., Madden, S. C., Galliano, F., Lebouteiller, V., Baes, M., Bendo, G. J., Boselli, A., Ciesla, L., Cormier, D., Cooray, A., Cortese, L., De Looze, I., Doublier-Pritchard, V., Galametz, M., Jones, A. P., Karczewski, O. Ł., Lu, N., Spinoglio, L., 2015. Linking dust emission to fundamental properties in galaxies: the low-metallicity picture. Astron. Astrophys. 582, A121.

Rouillé, G., Jäger, C., Krasnokutski, S. A., Krebsz, M., Henning, T., 2014. Cold condensation of dust in the ISM. Faraday Discuss. 168, 449.

Schneider, R., Hunt, L., Valiante, R., 2016. The dust content of the most metalpoor star-forming galaxies. Mon. Not. R. Astron. Soc. 457, 1842-1850.

Seok, J. Y., Koo, B.-C., Hirashita, H., Jul. 2015. Dust Cooling in Supernova Remnants in the Large Magellanic Cloud. Astrophys. J. 807, 100.

Silk, J., Rees, M. J., 1998. Quasars and galaxy formation. Astron. Astrophys. 331, L1-L4.

Smith, M. W. L., Gomez, H. L., Eales, S. A., Ciesla, L., Boselli, A., Cortese, L., Bendo, G. J., Baes, M., Bianchi, S., Clemens, M., Clements, D. L., Cooray, A. R., Davies, J. I., De Looze, I., di Serego Alighieri, S., Fritz, J., Gavazzi, G., Gear, W. K., Madden, S., Mentuch, E., Panuzzo, P., Pohlen, M., Spinoglio, L., Verstappen, J., Vlahakis, C., Wilson, C. D., Xilouris, E. M., 2012. The Herschel Reference Survey: Dust in Early-type Galaxies and across the Hubble Sequence. Astrophys. J. 748, 123.

Smith, R. J., Lucey, J. R., Hudson, M. J., 2009. Ages and metallicities for quiescent galaxies in the Shapley supercluster: driving parameters of the stellar populations. Mon. Not. R. Astron. Soc. 400, 1690-1705.

Su, Y., Irwin, J. A., 2013. Investigating the Potential Dilution of the Metal Content of Hot Gas in Early-type Galaxies by Accreted Cold Gas. Astrophys. J. 766, 61.

Temi, P., Brighenti, F., Mathews, W. G., 2007. Far-Infrared Spitzer Observations of Elliptical Galaxies: Evidence for Extended Diffuse Dust. Astrophys. J. 660, 1215-1231

Temi, P., Brighenti, F., Mathews, W. G., Bregman, J. D., 2004. Cold dust in early-type galaxies. i. observations. Astrophys. J. Suppl. 151, 237-269.

Tran, H. D., Tsvetanov, Z., Ford, H. C., Davies, J., Jaffe, W., van den Bosch, F. C., Rest, A., 2001. Dusty Nuclear Disks and Filaments in Early-Type Galaxies. Astron. J. 121, 2928-2942.

Tsai, J. C., Mathews, W. G., 1995. Interstellar Grains in Elliptical Galaxies: Grain Evolution. Astrophys. J. 448, 84.

Valentini, M., Brighenti, F., 2015. AGN-stimulated cooling of hot gas in elliptical galaxies. Mon. Not. R. Astron. Soc. 448, 1979-1998.

Valiante, R., Schneider, R., Salvadori, S., Bianchi, S., 2011. The origin of the dust in high-redshift quasars: the case of SDSS J1148+5251. Mon. Not. R. Astron. Soc. 416, 1916-1935. 
van Dokkum, P. G., Franx, M., 1995. Dust in the Cores of Early-Type Galaxies. Astron. J. 110, 2027.

Voit, G. M., Donahue, M., 1995. Problems with Cold Clouds and Cooling Flows. Astrophys. J. 452, 164.

Wagner, A. Y., Bicknell, G. V., 2011. Relativistic Jet Feedback in Evolving Galaxies. Astrophys. J. 728, 29.

Werner, N., Oonk, J. B. R., Sun, M., Nulsen, P. E. J., Allen, S. W., Canning, R. E. A., Simionescu, A., Hoffer, A., Connor, T., Donahue, M., Edge, A. C., Fabian, A. C., von der Linden, A., Reynolds, C. S., Ruszkowski, M., 2014. The origin of cold gas in giant elliptical galaxies and its role in fuelling radio-mode AGN feedback. Mon. Not. R. Astron. Soc. 439, 2291-2306.

Wiklind, T., Combes, F., Henkel, C., 1995. The molecular cloud content of early-type galaxies. V. CO in elliptical galaxies. Astron. Astrophys. 297, 643.

Zhukovska, S., Dobbs, C., Jenkins, E. B., Klessen, R. S., 2016. Modeling Dust Evolution in Galaxies with a Multiphase, Inhomogeneous ISM. Astrophys. J. 831, 147.

Zhukovska, S., Gail, H.-P., Trieloff, M., 2008. Evolution of interstellar dust and stardust in the solar neighbourhood. Astron. Astrophys. 479, 453-480. 